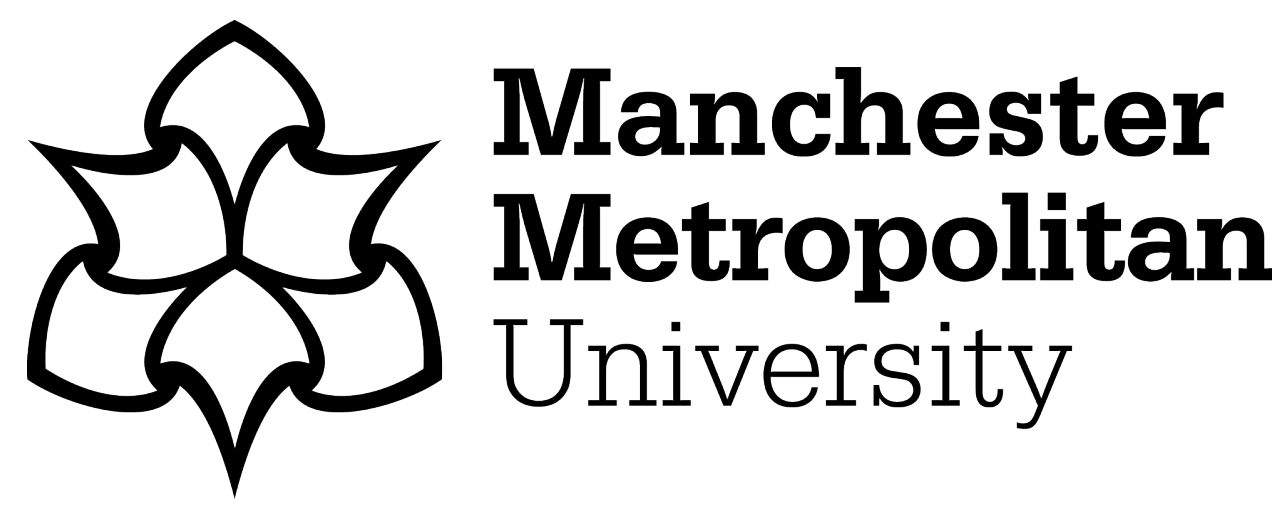

Cooper, R ORCID logoORCID: https://orcid.org/0000-0003-3370-5720 (2018) Socioeconomic adversity - an important barrier to healthy aging. BMJ (Online), 360. k1288-k1288. ISSN 0959-8146

Downloaded from: https://e-space.mmu.ac.uk/623726/

Version: Published Version

Publisher: BMJ Publishing Group

DOI: https://doi.org/10.1136/bmj.k1288

Usage rights: Creative Commons: Attribution 4.0

Please cite the published version 


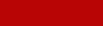

\title{
Socioeconomic adversity-an important barrier to healthy aging
}

\author{
Urgent action required to address socioeconomic inequalities in aging populations
}

\author{
Rachel Cooper senior lecturer \\ MRC Unit for Lifelong Health and Ageing at UCL, London WC1B 5JU, UK
}

As the global population ages there is concern that improvements in disability-free life expectancy might not have kept pace with the improvements in life expectancy that have driven this major demographic trend over the past century. This has important implications for individuals, families, and society; not least because it might prevent realisation of the potential opportunities of an aging population. ${ }^{1}$ Identifying the most effective targets for intervention to ensure that people live not only longer lives but also healthier, independent lives for longer is therefore an international research priority. ${ }^{2}$

It is within this context that, in a linked article (doi:10.1136/ bmj.k1046), Stringhini and colleagues have assessed the associations of low socioeconomic status and six other non-communicable disease risk factors with walking speed in later life. The authors selected these six other factors as they had been identified by the World Health Organization as targets for reducing premature mortality from chronic diseases by $25 \%$ by $2025 .{ }^{4}$ Walking speed—a quick, easy, and commonly used measure of physical capability-has two important strengths as an outcome. Firstly, it can be used to detect existing limitations in mobility ${ }^{5}$; a key threat to independent living. Secondly, slower walking speed is consistently associated with poorer health prospects, including lower survival rates and higher rates of incident disability in older community dwelling populations. $^{6-8}$

Previous research cited by Stringhini and colleagues has shown that lower socioeconomic status is associated with slower walking speed in older populations and that these associations extend back across life to childhood. By pooling results from 37 studies and 109107 adults aged 45-90 years from 24 countries, these comprehensive new analyses are able to compare the association of low socioeconomic status (usually based on last known occupation) and walking speed with associations with the six other WHO risk factors (obesity, smoking, high alcohol intake, physical inactivity, hypertension, and diabetes).

Using walking speed to estimate the number of years of physical functioning lost in association with each risk factor, the authors show that low socioeconomic status is at least as important as each of the six other factors. For example, in minimally adjusted models, estimated years of functioning lost by age 60 years among women were 4.6 (95\% confidence interval 3.6 to 6.2 ) for low socioeconomic status, 7.5 (6.1 to 9.5$)$ for obesity, 0.7 (0.1 to 1.5) for smoking, 0.1 ( -1.2 to 0.9$)$ for high alcohol intake, 5.4 (4.3 to 7.3) for physical inactivity, 3.0 (2.3 to 4.0) for hypertension, and 6.3 (4.9 to 8.4 ) for diabetes. These estimates varied markedly by sex and country; men and participants from high income countries lost more estimated years of functioning in relation to low socioeconomic status than women and those from low and middle income countries, respectively. However, the same general trend was found in all analyses.

Caution is required in interpreting these specific estimates: there is considerable heterogeneity within and between studies, and the analyses are cross sectional so observed differences in walking speed by age may also be attributable to cohort and period effects. Nevertheless, the main message of this paper is clear: socioeconomic adversity is an important target in global efforts to extend disability-free life expectancy. Furthermore, as socioeconomic status is more distal than the six other non-communicable disease risk factors studied, and these and other risk factors are socioeconomically patterned, there might be greater and more far reaching benefit in targeting socioeconomic adversity.

Another recent paper from the same research consortium provided compelling evidence of the need to target low socioeconomic status alongside other risk factors to reduce premature mortality. ${ }^{9}$ Taken together, these findings highlight the importance of socioeconomic adversity as a barrier to different key aspects of healthy aging. In comparing the findings of their two sets of analyses, ${ }^{39}$ the authors estimate that low socioeconomic status is associated with more years of physical functioning lost than years of life lost.

Although this is not surprising-previous research has documented wider socioeconomic inequalities in disability-free life expectancy than in overall life expectancy, ${ }^{10}$ it has important implications. The new findings suggest that people who have experienced socioeconomic adversity are not only more likely to live shorter lives but also to live more of their shorter lives with disability. Global increases in socioeconomic inequality ${ }^{11}$ 
are a cause for concern, and Stringhini and colleagues provide further evidence in support of renewed calls for action ${ }^{10} 12$ on inequality as a matter of urgency to improve the health and wellbeing of the aging global population.

With thanks to Diana Kuh for her helpful comments on an earlier draft of this editorial.

Competing interests: I have read and understood the BMJ policy on declaration of interests and declare the following: I am employed by UCL, the same institution as five of the co-authors of the research paper discussed, and receive research funding from the UK Medical Research Council (programme code: MC_UU_12019/4).

Provenance and peer review: Commissioned; not peer reviewed.

Foresight. Future of an ageing population. Government Office for Science, 2016. World Health Organization. World report on ageing and health. WHO, 2015.

3 Stringhini S, Carmeli C, Jokela M, etal . Socioeconomic status, non-communicable disease risk factors, and walking speed in older adults: multi-cohort population based study. $B M J$ 2018;360:k1046.
$4 \quad$ World Health Organization. Global action plan for the prevention and control of noncommunicable diseases 2013-2020. WHO, 2013.

5 Peel NM, Kuys SS, Klein K. Gait speed as a measure in geriatric assessment in clinical settings: a systematic review. J Gerontol A Biol Sci Med Sci 2013;68:39-46. 10.1093/gerona/gls174 22923430

6 Studenski S, Perera S, Patel K, etal . Gait speed and survival in older adults. JAMA 2011;305:50-8. 10.1001/jama.2010.1923 21205966

7 Cooper R, Kuh D, Hardy RMortality Review GroupFALCon and HALCyon Study Teams. Objectively measured physical capability levels and mortality: systematic review and meta-analysis. BMJ 2010;341:c4467. 10.1136/bmj.c4467 20829298

8 Perera S, Patel KV, Rosano C, etal . Gait speed predicts incident disability: A pooled analysis. J Gerontol A Biol Sci Med Sci 2016;71:63-71. 10.1093/gerona/glv126 26297942

9 Stringhini S, Carmeli C, Jokela M, etal. LIFEPATH consortium. Socioeconomic status and the $25 \times 25$ risk factors as determinants of premature mortality: a multicohort study and meta-analysis of 1.7 million men and women. Lancet 2017;389:1229-37. 10.1016/S0140-6736(16)32380-7 28159391

10 Marmot M. Social justice, epidemiology and health inequalities. Eur J Epidemiol 2017;32:537-46.28776115

11 OECD. In it together: Why less inequality benefits all. Paris, France: OECD Publishing 2015.

12 Tobias M. Social rank: a risk factor whose time has come? Lancet 2017;389:1172-4 10.1016/S0140-6736(17)30191-5 28159389

Published by the BMJ Publishing Group Limited. For permission to use (where not already granted under a licence) please go to http://group.bmj.com/group/rights-licensing/ permissions 